\title{
Biomarkers in mood disorders research: developing new and improved therapeutics
}

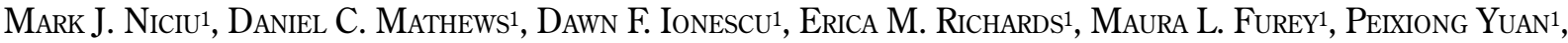 \\ Allison C. Nugent ${ }^{1}$, Ioline D. Henter 1 , Rodrigo Machado-Vieira ${ }^{1}$, Carlos A. Zarate Jr. ${ }^{1}$
}

'Experimental Therapeutics \& Pathophysiology Branch, Intramural Research Program, National Institute of Mental Health, National Institutes of Health, Bethesda, Maryland

Received: 11/9/14- Accepted: 15/9/14

DOI: 10.1590/0101-60830000000027

\begin{abstract}
Background: Recently, surrogate neurobiological biomarkers that correlate with target engagement and therapeutic response have been developed and tested in early phase studies of mood disorders. Objective: The identification of biomarkers could help develop personalized psychiatric treatments that may impact public health. Methods: These biomarkers, which are associated with clinical response post-treatment, can be directly validated using multimodal approaches including genetic tools, proteomics/metabolomics, peripheral measures, neuroimaging, biostatistical predictors, and clinical predictors. Results: To date, early phase biomarker studies have sought to identify measures that can serve as "biosignatures", or biological patterns of clinical response. These studies have also sought to identify clinical predictors and surrogate outcomes associated with pathophysiological domains consistently described in the National Institute of Mental Health's (NIMH) new Research Domain Criteria (RDoC). Using the N-methyl-D-aspartate (NMDA) antagonist ketamine as an example, we identified changes in several domains (clinical, cognitive, and neurophysiological) that predicted ketamine's rapid and sustained antidepressant effects in individuals with treatment-resistant major depressive disorder (MDD) or bipolar depression. Discussion: These approaches may ultimately provide clues into the neurobiology of psychiatric disorders and may have enormous impact Backon the development of novel therapeutics.
\end{abstract}

Niciu MJ et al. / Arch Clin Psychiatry. 2014;41(5):131-4

Keywords: Biomarker, depression, bipolar disorder, anxiety, drug development.

\section{Introduction}

In 2012, the United States Food and Drug Administration (FDA) approved 39 new drugs; however, none of these were approved for psychiatric indications ${ }^{1}$. While new and mechanistically distinct agents were approved for oncology and other medical subspecialties, negative results in early Phase II psychiatric research studies continue to limit the development and approval of successful compounds, despite the fact that psychiatric disorders have a greater morbidity burden than cardiovascular disease or cancer². Several factors have contributed to the proverbial drying of the medication pipeline, including high study attrition rates, long lead-time development, the heterogeneity of psychiatric disorders, and our lack of a mechanistic understanding of their underpinnings ${ }^{3}$. Thus, new and improved agents are urgently needed to treat psychiatric disorders; this, in turn, reinforces the need for additional research and investment for central nervous system (CNS) drug development. Our laboratory and others have focused on identifying specific biomarkers to tackle these challenges. Although larger collaborative efforts with industry will ultimately be required to validate these efforts, the identification and development of biomarkers by academia may kick-start drug development in psychiatric disorders.

\section{Background}

The NIH Biomarkers Definitions Working Group defines a "biomarker" as "a characteristic that is objectively measured and evaluated as an indicator of normal biological processes, pathogenic processes, or pharmacological responses to a therapeutic intervention 4 ". Researchers believe biomarkers to be particularly useful in three major areas: 1) drug development, 2) diagnosis and/or prognosis, and 3 ) patient management ${ }^{5}$. All three of these domains are directly involved in the personalized treatment of patients. Indeed, surrogate markers can hasten drug discovery by assessing early efficacy and reducing overall clinical trial duration and costs ${ }^{6}$. In psychiatry, and more specifically, in mood disorders, the search for potential biomarkers has encompassed several areas of research, including clinical/demographic factors, genetic/molecular factors, quantita- tive electroencephalography (qEEG), neuroimaging, and loudness dependence of auditory-evoked potentials ${ }^{7,8}$. As our burgeoning use of high-throughput "multi-omics" increases" (e.g., genomics, lipidomics, metabolomics, proteomics, and epigenetics), we will surely gain deeper insight into the pathophysiological mechanisms that underlie mood disorders.

In 2004, the FDA issued a "Critical Path Initiative" designed to increase the efficiency of product development and safety testing industry-wide. In 2005, the FDA also provided guidance for a voluntary submission path for initial, exploratory pharmacogenomic research data to improve biomarker-driven drug development. Notably, depression was considered a key therapeutic area for future studies and investment ${ }^{10}$. New techniques and disciplines in translational psychiatry also support the role of new therapeutic strategies. More recently, the model proposed by the National Institute of Mental Health (NIMH) new Research Domain Criteria (RDoC) reinforced the need to identify neural circuits and related biomarkers associated with psychiatric disorders, and detect early manifestations associated with increased risk for illness that may appear before the onset of cognitive and behavioral changes. The RDoC model also aims to define approaches centered on individual responses, as well as foster the augmented use of effective psychosocial approaches. This, in turn, will help reduce sample size and allow for researchers to generate more meaningful early proof-of-concept clinical trials and may help identify therapeutically relevant biomarkers.

\section{Biomarkers: qualification and exploration}

Validated biomarkers target objective measures of pathogenic or pharmacological effects. Notably, studies have found that such biomarkers reduce the risk of type I (false-positive) and type II (falsenegative) errors ${ }^{11}$. The process of biomarker qualification includes four broad concepts: exploration, demonstration, characterization, and surrogacy ${ }^{12}$. Wagner further defined proximal biomarkers as those identified in earlier phases of study - that is, those evaluating target engagement - and distal biomarkers as those characterized in later stages of investigation, typically in an illness pathway component ${ }^{12}$. On a separate but related note, the FDA has a Biomarkers 
Qualification Process (BQP) for biomarkers that are anticipated to affect regulatory review, drug development and, ultimately, patient care $^{13}$.

With regard to complex psychiatric disorders, current biomarker research has largely been circumscribed to the investigational phase; as a result, such work functions solely in the domains of research and development. Below, we review some of the most salient biomarker findings in psychiatric research.

Novel proof-of-concept studies ${ }^{14}$ from our laboratory have used clinical phenotypic data, neurophysiologic measures, peripheral and genetic markers and functional neuroimaging to not only help predict treatment outcomes with rapid-acting new antidepressants such as ketamine and scopolamine (though it should be noted that both are old pharmacological agents), but also to help clarify the underlying neural correlates of their well-described rapid antidepressant actions. For instance, elevated pretreatment/baseline anterior cingulate cortical activity in a fearful face paradigm predicted rapid antidepressant response to ketamine ${ }^{15}$. This result is consistent with previous findings that baseline anterior cingulate cortex (ACC) metabolism predicts antidepressant response to standard antidepressants ${ }^{16}$. The ketamine studies not only replicated previous findings but also expanded them to a novel non-monoaminergic drug whose antidepressant effects took place within a few hours. These findings strengthen the existing literature implicating a key role for ACC activity as a predictor for rapid antidepressant response ${ }^{17}$.

Another important finding is that of increased mammalian target of rapamycin (mTOR) phosphorylation in peripheral mononuclear cell protein after ketamine treatment ${ }^{18}$. These results concur with previous findings of rapid mTOR phosphorylation in the rat prefrontal cortex after low-dose ketamine in animal models of depression ${ }^{19}$. Because mTOR phosphorylation and associated signaling cascades have been involved in neoplastic processes and peripheral markers of mTOR activity (e.g., S6 kinase 1 (S6K1) inhibition) ${ }^{20}$, collecting such molecular profile data could offer insights into a hypothetical model to test other mTOR-active compounds for rapid antidepressant effects. Similarly, gathering data from patients at baseline could help identify potential responders before the drug (in this instance, ketamine) is even administered. While an a priori stratification approach has not been extensively performed in neuropsychiatric research, it is a rational conceptual framework for additional biomarker research in this field. One notable example is that the OPRM1A118G single nucleotide polymorphism (SNP) predicted antidepressant response to the opioid receptor antagonist naltrexone in alcohol use disorders ${ }^{21}$.

In addition to the benefits associated with identifying potential relevant therapeutic targets, early patient stratification could also lead to improved preclinical-to-clinical translation. With regard to major mood disorders, patients enrolled in a typical clinical trial can appear behaviorally similar (for instance, experiencing a major depressive disorder), but have different diagnoses (for instance, bipolar disorder vs MDD). In addition, the etiology of even the same mood disorder may differ from patient to patient, as may key genetic variables, consistent with the $\mathrm{RDoC}$ model (e.g., genes, molecules, cells, neural circuits, neurophysiology). As a result, only a subset of patients may respond to a specific molecular target. Initial patient stratification has been tested in medical specialties such as oncology; for instance, HER2 monoclonal antibody trastuzumab therapy in breast cancer tumors ${ }^{22}$. Cetuximab in epidermal growth factor receptor-overexpressing KRAS wild-type metastatic colorectal cancer used a similar approach ${ }^{23}$. Comparable methods have also been used to evaluate treatments for autoimmune diseases ${ }^{24,25}$.

Other advantages of patient stratification/enrichment include the improved design of treatment protocols; when used in early drug development, such protocols could study biomarkers at multiple levels (Figure 1). Also, the use of "efficacy-stratifying biomarkers ${ }^{14}$ " could influence patient data at the molecular level before initial treatment, thereby enhancing power and, by reducing heterogeneity, limiting the need for larger samples; a good example would be family history of alcohol use disorders in first-degree relatives of individuals with either treatment-resistant MDD26 or bipolar depression ${ }^{27}$. Such a translational model 28 in mood disorders may overcome heterogeneity challenges by excluding individuals at high risk of minimal response to a given intervention. Relatedly, collecting large databases of biological signatures/profiles would help identify and stratify targets for specific treatment approaches. This integrative method (Figure 1) could then be applied to other key agents to not only discover new therapeutics, but detect novel molecular targets and/or pathways that contribute to mood disorders.

\section{Diagnostic and prognostic biomarkers}

The development of diagnostic biomarkers for psychiatric disorders is a significant challenge, particularly because psychiatric disorders are typically characterized based on clinical observations of behavioral changes. However, the development of neurobiologically-informed diagnostic biomarkers represents a key area for further investigation in psychiatric research. Specifically, identifying diagnostic biomarkers is not based on current descriptive (and, as some have argued, theoretical) or categorical frameworks but, instead, is based on alternative dimensional concepts of mental disorders, such as the psychiatric framework established by the RDoC ${ }^{29}$. Furthermore, while initial studies have been conducted with multi-assay serumbased diagnostic tests for $\mathrm{MDD}^{30}$, multimodal biomarker panels of pharmacologic and/or safety/toxicity profiles may ultimately represent a more valuable tool for both psychiatric research and subsequently, clinical practice.

In addition, identifying predictive biomarkers in mood disorders using a "systems biology"-based approach that collects and integrates several hierarchical levels or domains ${ }^{31}$ is a promising tool for future research. This integrative approach - which can include molecular data, structural and functional neuroimaging, and clinical correlates - contrasts with paradigms established by previous scientific discoveries. For example, both glial and synaptic dysfunction have been implicated in the pathophysiology of mood disorders ${ }^{32}$; however, it is unlikely that they could be considered a sole causal factor Rather, the dynamic interplay between neuronal and non-neuronal biological mechanisms at the genetic, molecular, or cellular level may generate the dysfunctional neural circuitry and decreased cellular resilience associated with mood disorders ${ }^{33}$. Supporting this model, the calcium channel, voltage-dependent, L type alpha $1 \mathrm{C}$ subunit gene (CACNA1C) was found to be involved in several psychiatric disorders $^{34}$, though it remains unclear whether the associated molecular changes were a cause or an effect of the dysfunction in upstream or downstream domains, or what their key functional targets impacting behavioral and cognitive modulation might be. In this context, a "systems biology" method has been effectively exploited in oncology and other medical areas, where heterogeneity is usually present in diseases subtypes. This innovative "systems biology" approach remains untested for the study of neuropsychiatric diseases. Devoted interdisciplinary approaches will be required in order to accumulate sufficient quantitative data and develop new computational neuroscience models and other innovative approaches before its full potential can be assessed ${ }^{35,36}$.

\section{Limitations and future directions}

Despite the great potential associated with developing clinically useful biomarkers in personalized medicine, and the excitement over this burgeoning field, fewer than $100 / 150,000(0.06 \%)$ publications in the biomedical biomarker literature have been endorsed for routine clinical practice ${ }^{37}$. Questions regarding standardization of specimen collection/storage, matching control and disease samples (methods and size), and replication difficulties continue to limit the development of potential translational findings into clinical practice ${ }^{37}$. In addition, close, careful follow-up with statistical and computational specialists, as well as data sharing networks and agreements, will be needed to draw definitive conclusions regarding biomarker research in large datasets. In this context, imaging initiatives such as the International Neuroinformatics Coordinating Facility (INCF) 


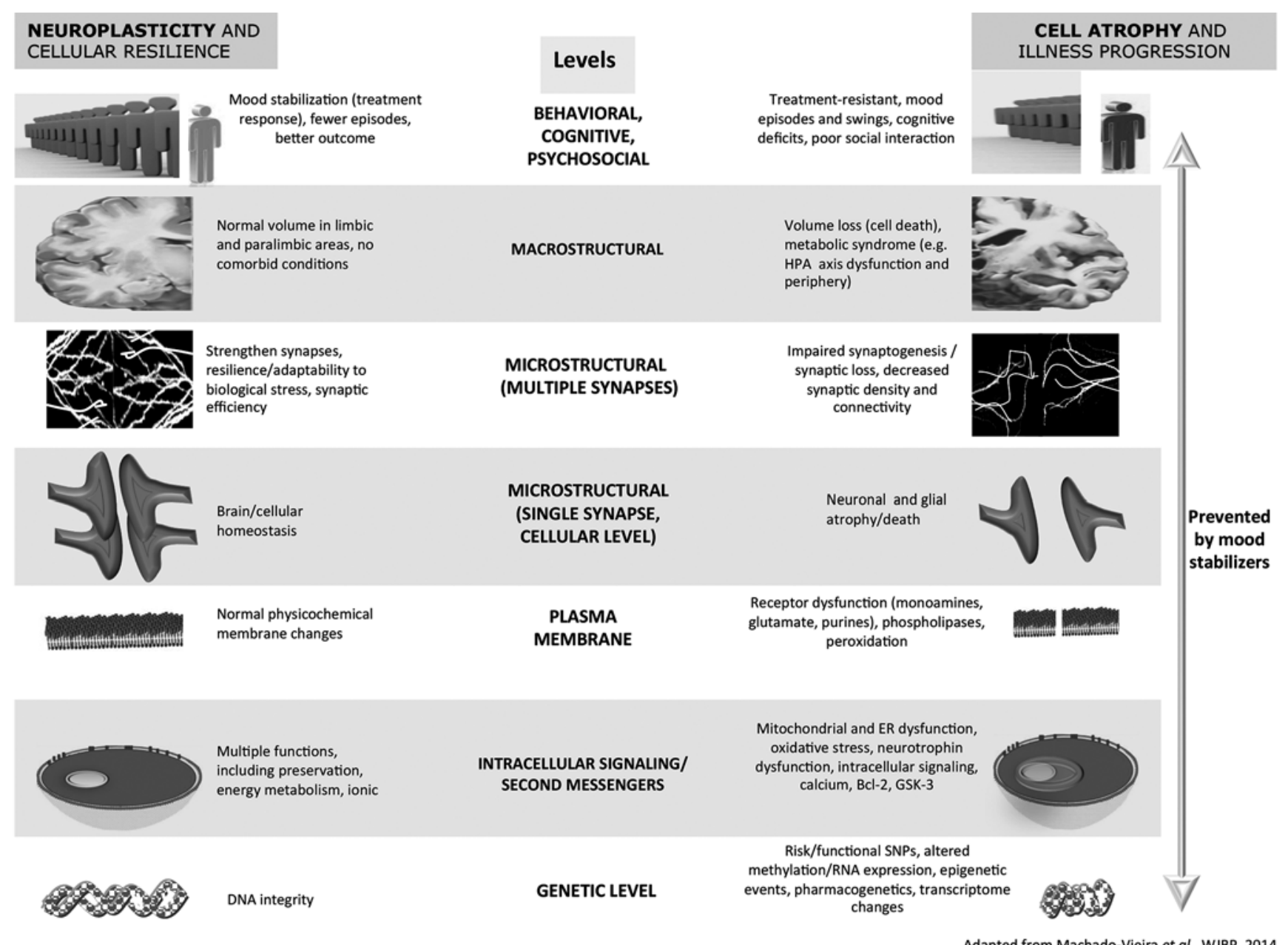

Figure 1. Biomarkers in psychiatry: functional and pathological findings and their potential therapeutic relevance. HPA: hypothalamic-pituitary-adrenal; Bcl-2: B-cell lymphoma 2; GSK-3: glycogen synthase kinase 3; SNP: single nucleotide polymorphism.

and their computing platforms (e.g., the NeuroDebian project) may allow for more efficient collaboration between academia, government, and private initiatives ${ }^{38}$. These types of collaboration focus on promoting collaborative networks instead of the more typical isolated investigator-driven approach ${ }^{37}$.

In the current "translational" era, all knowledge tends to be merged; multiple efforts work to reach a broad common goal: the progress of science. Because of the current limitations of diagnostic and treatment specificity, however, it is unlikely that any currently identified individual biomarker will substantially impact neuropsychiatric research or care. Also, the development of biomarkers in a cost-effective manner will be challenging ${ }^{39}$, particularly because it will require new insights into systems biology and the development of other bioinformatics approaches ${ }^{40}$. Relatedly, it will be crucial to address the heterogeneity of descriptive diagnoses and evaluate neurobiologically-defined subgroups for maximal efficacy, particularly because fewer drugs have been approved for neuropsychiatric indications in the last few years. Other challenges that will need to be addressed include the development of new diagnostic tools and scoring systems for quantitative biomarkers, and the development of clinical predictors of response and surrogate outcomes. With regard to bipolar disorder in particular, several courses of action might be taken to improve the success rates of clinical research while saving both time and money, for instance, conducting small proof-ofconcept Phase 1 trials of novel agents in surrogate populations (e.g. subjects with hyperthymia instead of bipolar disorder), or using pharmacological agents able to mimic some of the disease features in healthy volunteers. It is equally important to design clinical trials that can estimate the chances of success in initial clinical development.

As discussed above, the focus in drug development has recently changed from hypothesis-driven studies to hypothesis-generating experiments using the new "-omics" generation of platforms and techniques. Though these technologies allow for unprecedented exploration of the mechanisms of action underlying various disorders, their results are much broader and can thus be difficult to interpret. For instance, $80 \%$ of the 25,000 human genes have some effect on the brain. Thus, hypothesis-generating approaches may even increase the level of intricacy associated with such studies due to their inability to "sift the wheat from the chaff".

Finally, there is also a serious need to "post-translate" results from proof-of-concept clinical research into medical care ${ }^{41}$. Such "translation" is imperative before scientific discoveries can be transformed from preclinical research into clinical applications, thus closing the gap between drug discovery in preclinical models and drug development in humans. Using new technologies to conduct close, careful identification of new biomarkers - those associated with clinical relevance or prediction of pharmacological response to a particular treatment - will ensure more accurate individual treatment for those who need it most.

\section{Acknowledgements and disclosures}

The authors gratefully acknowledge the support and funding of the Intramural Research Program at the National Institute of Mental Health, National Institutes of Health (IRP-NIMH-NIH; NCT num- 
ber: NCT00088699). Dr. Zarate was also supported by a NARSAD Independent Investigator Award from the Brain and Behavior Research Foundation.

Dr. Zarate is listed as a coinventor on a patent for the use of ketamine and its metabolites in major depression. Dr. Zarate has assigned his rights in the patent to the US government but will share a percentage of any royalties that may be received by the government. All other authors have no conflict of interest to disclose, financial or otherwise.

\section{References}

1. Mullard A. 2012 FDA drug approvals. Nat Rev Drug Discov. 2013;12(2):87-90.

2. Wittchen HU, Jacobi F, Rehm J, Gustavsson A, Svensson M, Jonsson B, et al. The size and burden of mental disorders and other disorders of the brain in Europe 2010. Eur Neuropsychopharmacol. 2011;21(9):655-79.

3. Miller G. Is pharma running out of brainy ideas? Science. 2010;329(5991):502-4.

4. Biomarkers Definitions Working Group. Biomarkers and surrogate endpoints: preferred definitions and conceptual framework. Clin Pharmacol Ther. 2001;69(3):89-95.

5. Hayes RL, Robinson G, Muller U, Wang KK. Translation of neurological biomarkers to clinically relevant platforms. Methods Mol Biol. 2009;566:303-13.

6. Staner L. Surrogate outcomes in neurology, psychiatry, and psychopharmacology. Dialogues Clin Neurosci. 2006;8(3):345-52.

7. Papakostas GI. Surrogate markers of treatment outcome in major depressive disorder. Int J Neuropsychopharmacol. 2012;15(6):841-54.

8. Phillips ML, Vieta E. Identifying functional neuroimaging biomarkers of bipolar disorder: toward DSM-V. Schizophr Bull. 2007;33(4):893-904.

9. Schwarz E, Bahn S. The utility of biomarker discovery approaches for the detection of disease mechanisms in psychiatric disorders. Br J Pharmacol. 2008;153(Suppl 1):S133-6.

10. Amur S, Frueh FW, Lesko LJ, Huang SM. Integration and use of biomarkers in drug development, regulation and clinical practice: a US regulatory perspective. Biomark Med. 2008;2(3):305-11.

11. Karsdal MA, Henriksen K, Leeming DJ, Mitchell P, Duffin K, Barascuk $\mathrm{N}$, et al. Biochemical markers and the FDA Critical Path: how biomarkers may contribute to the understanding of pathophysiology and provide unique and necessary tools for drug development. Biomarkers. 2009;14(3):181-202.

12. Wagner JA. Strategic approach to fit-for-purpose biomarkers in drug development. Annu Rev Pharmacol Toxicol. 2008;48:631-51.

13. Goodsaid FM, Mendrick DL. Translational medicine and the value of biomarker qualification. Sci Transl Med. 2010;2(47):47ps44.

14. Zarate CA Jr., Mathews DC, Furey ML. Human biomarkers of rapid antidepressant effects. Biol Psychiatry. 2013;73(12):1142-55.

15. Salvadore G, Cornwell BR, Colon-Rosario V, Coppola R, Grillon C, Zarate $\mathrm{CA}$ Jr., et al. Increased anterior cingulate cortical activity in response to fearful faces: a neurophysiological biomarker that predicts rapid antidepressant response to ketamine. Biol Psychiatry. 2009;65(4):289-95.

16. Mayberg HS, Brannan SK, Mahurin RK, Jerabek PA, Brickman JS, Tekell $\mathrm{JL}$, et al. Cingulate function in depression: a potential predictor of treatment response. Neuroreport. 1997;8(4):1057-61.

17. Pizzagalli DA. Frontocingulate dysfunction in depression: toward biomarkers of treatment response. Neuropsychopharmacology. 2011;36(1):183-206.

18. Denk MC, Rewerts C, Holsboer F, Erhardt-Lehmann A, Turck CW. Monitoring ketamine treatment response in a depressed patient via peripheral mammalian target of rapamycin activation. Am J Psychiatry. 2011;168(7):751-2.
19. Li N, Lee B, Liu RJ, Banasr M, Dwyer JM, Iwata M, et al. mTOR-dependent synapse formation underlies the rapid antidepressant effects of NMDA antagonists. Science. 2010;329(5994):959-64.

20. Hoeffer CA, Klann E. mTOR signaling: at the crossroads of plasticity, memory and disease. Trends Neurosci. 2010;33(2):67-75.

21. Arias AJ, Sewell RA. Pharmacogenetically driven treatments for alcoholism: are we there yet? CNS Drugs. 2012;26(6):461-76.

22. Mass RD, Press MF, Anderson S, Cobleigh MA, Vogel CL, Dybdal N, et al. Evaluation of clinical outcomes according to HER2 detection by fluorescence in situ hybridization in women with metastatic breast cancer treated with trastuzumab. Clin Breast Cancer. 2005;6(3):240-6.

23. Karapetis CS, Khambata-Ford S, Jonker DJ, O'Callaghan CJ, Tu D, Tebbutt NC, et al. K-ras mutations and benefit from cetuximab in advanced colorectal cancer. N Engl J Med. 2008;359(17):1757-65.

24. Han NY, Kim EH, Choi J, Lee H, Hahm KB. Quantitative proteomic approaches in biomarker discovery of inflammatory bowel disease. J Dig Dis. 2012;13(10):497-503.

25. Lewis JD. The utility of biomarkers in the diagnosis and therapy of inflammatory bowel disease. Gastroenterology. 2011;140(6):1817-26.

26. Phelps LE, Brutsche N, Moral JR, Luckenbaugh DA, Manji HK, Zarate CA Jr. Family history of alcohol dependence and initial antidepressant response to an N-methyl-D-aspartate antagonist. Biol Psychiatry. 2009;65(2):181-4

27. Luckenbaugh DA, Ibrahim L, Brutsche N, Franco-Chaves J, Mathews D, Marquardt CA, et al. Family history of alcohol dependence and antidepressant response to an $\mathrm{N}$-methyl-D-aspartate antagonist in bipolar depression. Bipolar Disord. 2012;14(8):880-7.

28. Laifenfeld D, Drubin DA, Catlett NL, Park JS, Van Hooser AA, Frushour $\mathrm{BP}$, et al. Early patient stratification and predictive biomarkers in drug discovery and development: a case study of ulcerative colitis anti-TNF therapy. Adv Exp Med Biol. 2012;736:645-53.

29. Insel T, Cuthbert B, Garvey M, Heinssen R, Pine DS, Quinn K, et al. Research domain criteria (RDoC): toward a new classification framework for research on mental disorders. Am J Psychiatry. 2010;167(7):748-51.

30. Papakostas GI, Shelton RC, Kinrys G, Henry ME, Bakow BR, Lipkin SH, et al. Assessment of a multi-assay, serum-based biological diagnostic test for major depressive disorder: a pilot and replication study. Mol Psychiatry. 2013;18(3):332-9.

31. McIntosh AM. Toward a systems biology of mood disorder. Biol Psychiatry. 2013;73(2):107-8.

32. Machado-Vieira R, Manji HK, Zarate CA. The role of the tripartite glutamatergic synapse in the pathophysiology and therapeutics of mood disorders. Neuroscientist. 2009;15(5):525-39.

33. Iris F. Psychiatric systems medicine: closer at hand than anticipated but not with the expected portrait. Pharmacopsychiatry. 2012;45(Suppl 1):S12-21.

34. Cross-Disorder Group of the Psychiatric Genomics Consortium. Identification of risk loci with shared effects on five major psychiatric disorders: a genome-wide analysis. Lancet. 2013;381(9875):1371-9.

35. Kitano H. Systems biology: a brief overview. Science. 2002;295(5560):1662-4

36. Hoffman RE, Grasemann U, Gueorguieva R, Quinlan D, Lane D, Miikkulainen R. Using computational patients to evaluate illness mechanisms in schizophrenia. Biol Psychiatry. 2011;69(10):997-1005.

37. Poste G. Bring on the biomarkers. Nature. 2011;469(7329):156-7.

38. Poline JB, Breeze JL, Ghosh S, Gorgolewski K, Halchenko YO, Hanke M, et al. Data sharing in neuroimaging research. Front Neuroinform. 2012;6:9.

39. Boksa P. A way forward for research on biomarkers for psychiatric disorders. J Psychiatry Neurosci. 2013;38(2):75-7.

40. Kobeissy F, Alawieh A, Mondello S, Boustany RM, Gold MS. Biomarkers in psychiatry: how close are we? Front Psychiatry. 2013;3:114.

41. Machado-Vieira R. Tracking the impact of translational research in psychiatry: state of the art and perspectives. J Transl Med. 2012;10:175. 


\title{
Anorexia nervosa, paternalism and clinical practice
}

\author{
Gustavo Costa Medeiros ${ }^{1}$, Daniela Guimarães Sampaio ${ }^{1}$, Felipe Corchs ${ }^{1}$ \\ 1 University of São Paulo Medical School (FMUSP), Department and Institute of Psychiatry, São Paulo, SP, Brazil.
}

Received: 7/10/2014 - Accepted: 7/23/2014

DOl: 10.1590/0101-60830000000028

\section{Dear Editor}

Anorexia nervosa (AN) is an eating disorder whose therapeutic interventions usually produce unsatisfactory results. This disorder is characterized by high rates of relapse, tendency to chronicity, high morbidity and mortality ${ }^{1}$. Low motivation is a central issue that hinders $\mathrm{AN}$ treatment ${ }^{1}$. Interpersonal relationships seem to be crucial in AN and may play a role in this sense as well. We propose that specific patterns of interpersonal interactions can arise when professionals are dealing with AN subjects and that this peculiar rapport is not only a characteristic of the disorder in question, but could also be a part of what perpetuates and aggravates the problem.

Those who interact with AN patients can refer feelings of helplessness, fear, anxiety and hopelessness ${ }^{2}$, pointing to a possible degree of aversivity in these relationships. This fact may be aggravated by the slow improvement typical of AN, which can be frustrating and, as a result of this, professionals can adopt a strong and directive attitude toward the patients trying to reach better outcomes in the treatment. In fact, a coercive attitude in AN can generate relatively quick results ${ }^{3}$, what can provide a transient attenuation of professional's discomfort. This may have reinforcing effects over the controlling attitude, making it more likely to occur in future similar occasions. In other words, too much directivity appears to relief professional's distress and, therefore, negatively reinforce coercive posture, but it may also have adverse consequences to the patient's treatment ${ }^{4}$.

Fallouts are noticed as a remarkably paternalistic approach seen, for example, in procedures such as involuntary hospitalization not based on scientific guideless or arbitrary tube feeding - tends to increase the already high resistance in these patients ${ }^{5}$. An intimidating posture also can punish potentially unpleasant statements made by the patient, possibly suppressing genuine reports and predisposing to false descriptions of improvement. Coercion-generated improvements may be volatile and fragile and even unfavorable in the long term. For instance, forced hospitalizations decrease the voluntary search of treatment ${ }^{3}$, which is a major issue since the motivation to change is an important element for therapeutic success ${ }^{1}$.
A healthy rapport seems to have an important role in reaching a favorable outcome in treatments since a mutual and reliable alliance in $\mathrm{AN}$ increases the chance of a continuous and more effective approach ${ }^{4}$. However, according to Darcy et al. ${ }^{6}$ the therapist-patient alliance is yet relatively under-researched especially when studied from the professional perspective and there is a deficiency of mental health professional's continued training on the use of rational paternalistic techniques ${ }^{3}$.

In short, some evidence suggests that a directive posture is more common in AN compared to other mental disorders and may have unfavorable effects over its treatment. It seems to be important to establish a positive therapeutic alliance to optimize motivation in this disorder. Prioritize cooperation in AN apparently can shifts the focus from control to an efficient therapeutic bond. Further research on the professional-patient relationship in AN cases is needed in other to elucidated this point and enhance the success of interventions.

\section{References}

1. Espíndola CR, Blay SL. Long term remission of anorexia nervosa: factors involved in the outcome of female patients. PLoS One. 2013;8(2):e56275.

2. Gabbard GO. Chapter 12 - Substances related disorders and eating disorders. In: Gabbard GO. Psychodynamic psychiatry in clinical practice. $4^{\text {th }}$ ed. Washington, DC: American Psychiatric Publishing; 2006. p. 259-82.

3. Seo MK, Kim SH, Kyu RM. Coercion in psychiatric care: can paternalism justify coercion? Int J Soc Psychiatry. 2013;59(3):217-23.

4. Horvath AO, Del Re AC, Flückiger C, Symonds D. Alliance in individual psychotherapy. Psychotherapy (Chic). 2011;48(1):9-16.

5. Bischoff MM, Terence JG. The client resistance predicted by therapist behavior: a study of sequential dependence. J Couns Psychol. 1995;42(4):487-95.

6. Darcy AM, Katz S, Fitzpatrick KK, Forsberg S, Utzinger L, Lock J. All better? How former anorexia nervosa patients define recovery and engaged in treatment. Eur Eat Disord Rev. 2010;18(4):260-70. 